\title{
Les interactions en ligne et la construction du savoir dans les dispositifs de formation complètement à distance : Quels rapports ?
}

\section{Online interactions and the construction of knowledge in completely remote training systems: What are the relationships?}

\author{
Meryem Mouni ${ }^{{ }^{*}}$ and Abdallah Boumalek ${ }^{1}$ \\ ${ }^{1}$ Laboratory CEDUC, UMP, Faculty of arts and humanities, Oujda, Morocco
}

\begin{abstract}
Résumé. Cette contribution se propose d'étudier la relation entre les interactions en ligne et la construction collaborative du savoir dans trois MOOC. Son objectif principal est l'examen, dans l'ingénierie pédagogique adoptée, du rôle envisagé pour les interactions en ligne dans le processus d'apprentissage. Elle tentera également de comprendre comment les forums de discussion contribuent à une co-construction des savoirs entre les participants. Le modèle d'analyse des interactions adopté dans ce travail est l'analyse conversationnelle d'inspiration ethnométhodologique qui renvoie aux actions. Elle étudie les conversations dans un espace écologique et tâche de comprendre leur organisation séquentielle. Ce modèle est foncièrement centré sur les interactions, en vue de décrire les stratégies adoptées par les participants présents lors des tours de paroles. Dans cette perspective, ce travail n'ambitionne pas l'étude des aspects iconographiques que véhiculent les interactions en ligne. Le corpus d'étude est composé de forums de discussion enregistrés sur des $\mathrm{MOOC}$ dans le but de répondre à notre objectif de recherche.
\end{abstract}

\begin{abstract}
This contribution aims to study the link between online interactions and the collaborative construction of knowledge in three MOOCs. Its main objective is to examine, in the chosen educational engineering, the role envisaged for online interactions in the learning process. We will also try to understand how discussion forums contribute to a co-construction of knowledge between participants. The interaction analysis model adopted in this work is conversational ethno-methodological analysis that refers to actions. She studies the conversations taking place in an ecological space and tries to understand their sequential organization. This model is fundamentally centred on interactions, in order to describe the strategies adopted by the participants present during the speaking tours. In
\end{abstract}

* Corresponding author: mouni.meryem@gmail.com 
this perspective, this work does not study the iconographic aspects conveyed by online interactions. The study corpus is made up of discussion forums recorded on MOOCs in order to meet three research objectives

\section{Introduction}

L'e-learning utilise des méthodes dites actives pour répondre à la nature de l'enseignement et à sa mission; il s'agit d'une structure dans laquelle enseignants et étudiants ne sont pas réellement regroupés dans la salle de classe, mais la structure virtuelle l'est en réalité. Cet enseignement intègre les considérations nécessaires à l'interaction entre les différents acteurs du système et propose des forums de discussion comme méthode alternative pour la gestion de l'interaction entre les apprenants en ligne. Cependant, le travail de groupe ou collectif, la collaboration, la coopération et la construction sont des concepts utilisés depuis longtemps. Depuis de nombreuses années déjà, le travail collaboratif entre apprenants fait l'objet de recherches pédagogiques. Le problème à résoudre concerne l'attribution des tâches, le rôle de l'apprenant et les postures prises par l'enseignant lorsqu'il travaille avec un groupe. L'interaction entre les apprenants en groupe est cruciale. L'enseignant est intéressé par la communication entre les apprenants en petits groupe de travail. Ils acquerront également ces compétences en communication et les relations interpersonnelles qu'ils ont établies. De même, les apprenants interagissent entre eux et effectuent les activités proposées par l'enseignant. Ce type d'interaction entre apprenants est considéré comme une sorte d'interaction sociale, qui peut cultiver les compétences sociales des apprenants et leur permettre de mieux s'intégrer dans la société. "C'est cette vision de l'apprentissage participatif qui est au cour de la perspective actionnelle telle qu'envisagée par le Cadre européen commun de référence pour les langues apprendre, enseigner, évaluer [1]."

La perspective actionnelle est une réponse à l'approche communicative qui réduit la communication dans la situation de classe simulée. D'un autre côté, l'apprenant a un rôle social du point de vue de l'action; il mobilise ce qu'il a appris en classe en réalisant de vraies activités dans la vie sociale. L'interaction sociale dans l'apprentissage est au cœur de plusieurs théories et recherches, en particulier celles privilégiant la dimension sociale de l'apprentissage comme Vygotsky et ses successeurs qui considèrent l'apprentissage collectif comme un moyen de construction de connaissances.

Aujourd'hui, l'interaction ne se produit pas seulement en face à face. Avec le développement de la technologie numérique, les enseignants et les apprenants ont commencé à reconsidérer le concept de travail en groupe. Grâce à des dispositifs d'enseignement ouvert et à distance, l'apprentissage est sujet à des changements profonds. De nouveaux outils de communication et d'interaction sont en cours de création. Ces outils peuvent promouvoir le travail collaboratif et l'interaction sociale en ligne entre les apprenants. Le travail de groupe en ligne estégalement devenu l'objet de recherches et de discussions, révélant de nouveaux domaines de recherche [2].

\section{Contexte et problématique}

“Aujourd'hui, les MOOC (Massive Open Online Courses) prennent de plus en plus le devant de l'environnement éducatif international. Si ces cours en ligne redonnent à la formation à distance une notoriété qui lui faisait jusque-là défaut, ils sont aussi les prémices d'une révolution dans les milieux académiques. Les universités sont amenées à construire des stratégies pour gagner leur autonomie et trouver leur place dans la mondialisation, parmi ces stratégies, les technologies numériques sont devenues incontournables. Celles-ci ne se résument plus à un volet additionnel porté par les services 
TICE, mais elles sont des leviers pour l'ensemble du projet d'établissement [3]. "Toutefois, le succès de ces plateformes « massivement ouvertes » est encore loin d'être assuré, leur financement et leur efficacité étant relativement incertains. Par ailleurs, ces cours en ligne ne délivrent que des certificats dont la valeur sur le marché de l'emploi est pour le moment limitée. Il est donc difficile de prédire quelle forme prendra l'Université de demain. Mais une chose est sûre, le virage initié par les MOOC ouvre de nouvelles perspectives en pédagogie universitaire [3].

Nous visons, par la présente étude, l'analyse des interactions sociales en ligne au sein des espaces d'échanges (Forum). Nous étudions les interactions entre les apprenants qui collaborent entre eux en vue de la réalisation des tâches demandées. Il s'agit pour nous de déterminer dans quelle mesure ces outils de collaboration peuvent permettre une coconstruction entre les apprenants dans les dispositifs de formation complètement à distance: trois MOOC dans notre cas. Notre recherche se situe donc dans cette perspective actionnelle où l'apprenant se voit comme un acteur social qui réalise des tâches en collaboration avec d'autres apprenants, tout en développant à la fois des habiletés cognitives, sociocognitives et socio-affectives. Nous nous intéressons plus particulièrement aux manifestations de la Co-construction dans ce genre d'espaces en ligne. Pour ce faire, nous avons adopté une observation participante pour visualiser la traçabilité des apprenants tout au long de la formation. Ensuite, une méthode qualitative en vue d'examiner les forums de discussions des trois MOOC que nous avons choisis pour notre étude, à savoir : MOOC 1: "What's up Doc-Formation documentaire pour tous ", MOOC 2 : «Préparer et réussir le DELF B2 et le DALF C1》, MOOC 3 : «Éducation par la recherche : smart city à l'École ». Pour le cas des forums, nous adopterons un modèle d'analyse conversationnelle d'inspiration ethnométhodologique sur la base des travaux de deux chercheurs : Michel Marcoccia [4] et Lorenza Mondada [5]. Nous avons donc voulu déceler les aspects et les manifestations de la Co-construction dans ces espaces de collaboration en ligne. Ainsi, nous avons formulé trois questions de recherche :

1. Comment la collaboration entre apprenants dans les dispositifs de formation en ligne est-elle envisagée?

2. Jusqu'à quel point les forums peuvent-ils faciliter la collaboration et la communication entre les participants (étudiants universitaires) dans un dispositif de formation en ligne?

3. Quel est le rôle des interactions asynchrones en ligne dans la construction collaborative des savoirs dans les formations enligne?

L'analyse des interactions en ligne se veut actuellement comme un champ disciplinaire autonome qui trouve toute sa légitimité dans son apport aux études portant sur des apprentissages médiatisés en milieu universitaire [6].

Les assises théoriques qui nourrissent ce champ d'analyse sont diverses. Citons-en ici les principales : l'interactionnisme symbolique selon Blumer [7], l'ethnométhodologie selon Garfinkel [8], les sciences humaines selon Kerbrat-Orecchioni [9]; Vion [10], la psychologie de l'apprentissage selon Perret-Clermont [11], l'anthropologie culturelle selon Lave [12], en plus des travaux sur l'analyse du discours et l'analyse conversationnelle que nous adoptons comme modèle d'analyse dans ce travail.

L'étude menée dans le cadre de cet article s'inscrit dans la continuité des travaux qui traitent la question des interactions en ligne, notamment l'analyse conversationnelle. Elle essaiera de répondre à la problématique suivante :

Dans quelle mesure les interactions pédagogiques en ligne permettent-elles la construction collaborative des savoirs sur les dispositifs de formationcomplètement en ligne ?

Seront alors formulées trois hypothèses de recherche qui nous aideront à apporter des éléments de réponse à notre problématique.

1. L'adaptation d'une ingénierie pédagogique appropriée favorise les interactions 
en ligne au service de la co-constructiondu savoir.

2. Les forums représentent un moyen adéquat de collaboration et de production à distance.

3. Les interactions asynchrones contribuent de manière intelligible à la coconstruction du savoir.

\section{Choix méthodologiques (analyse conversationnelle choix ethnomethodologique)}

L'analyse conversationnelle [4]-[13] peut mettre en évidence certains phénomènes liés à la dynamique de la conversation. Le travail dans ce cadre est généralement basé sur une méthode qui consiste à appliquer les catégories classiques de l'analyse de conversation aux échanges assistés par ordinateur pour en identifier les particularités. En fait, cette méthode peut permettre aux analystes d'observer les limites possibles des types d'analyse utilisés, non seulement pour l'apprentissage en ligne, mais aussi pour de nombreuses formes non développées de communication assistée par ordinateur. En somme, l'apprentissage en ligne peut révéler des problèmes qui existent encore dans le domaine de l'analyse du dialogue.

Les paramètres jugés nécessaires pour définir une conversation ne sont pas tous bien adaptés pour classer les interactions lors de l'apprentissage en ligne. Lorsque les étudiants participants entrent et sortent de l'espace de communication la discussion est relativement dispersée. Par conséquent, l'utilisation de méthodes d'analyse de conversation peut mieux décrire et comprendre la dynamique de la communication dans le système de formation en ligne, et peut également remettre en question ces méthodes, montrer leurs limites et proposer des améliorations [13].

\section{Recueil des données sur les forums}

Un forum est un espace de discussion dédié à tous (ou du moins ouvert à quelques participants). Les discussions qui y sont stockées permettent une communication asynchrone (c'est ce qui distingue les forums de la messagerie instantanée) [14]. Il existe deux types de forums, selon le type de message : soit un forum de discussion qui catégorise les messages par ordre chronologique, soit un forum de questions / réponses qui catégorise les messages par vote.

Les interactions sur les forums présentent un avantage majeur : elles sont archivées et leurs traces conservées, ce qui permet leur visualisation à tout moment. Nous signalons que les interactions se sont produites d'une manière écologique avant d'être recueilliespar le chercheur. L'analyse conversationnelle en fait, s'intéresse aux conversations 'naturelles' qui ne sont pas provoquées par le chercheur. De ce fait, notre corpus est constitué de données créées naturellement dans leur contexte sans aucune intervention ou perturbation de ces interactions de notre part. Les données en question nécessitent naturellement une sélection bien précise et raisonnée pour la constitution d'un 'échantillon' représentatif sur lequel nous nous appuierons pour apporter des éléments de réponses à nos hypothèses ainsi qu'aux questions de recherche soulevées.

Dès lors, une étude de notre contexte s'avère importante. Nous tenons tout d'abord à rappeler que l'étude s'intéresse en grande partie aux interactions dans les forums de discussion entre les participants dans le cadre de trois MOOC, les jours accordés pour les échanges varient d'une semaine à l'autre. Quelquefois, il s'agit de moins d'une semaine, d'autres davantage. 


\section{Présentation des mooc}

Le MOOC « What's up Doc - Formation documentaire pour tous » vise à faire maîtriser aux participants les étapes d'une recherche documentaire. Autrement dit, il leur permet de trouver et d'exploiter des documents de qualité en fonction de leurs besoins d'information.

What's up Doc vise un usage documentaire dans une démarche de travail académique ou universitaire qui a ses exigences particulières : une recherche universitaire n'est pas un travail journalistique, elle s'appuie sur de la documentation scientifique (qu'elle soit du domaine des sciences humaines, des sciences exactes, appliquées ou de la santé) et sur une méthode d'analyse. Cependant, ce cours n'est pas un guide des ressources documentaires dans les différentes disciplines du savoir. Cela est un vaste projet qui dépasse de loin nos ambitions, mais qui pourrait faire l'objet d'un autre cours. Il existe de multiples répertoires de ressources sur le net, nous en évoquerons certains afin de mener des recherches dans de vrais outils documentaires. Et puis, si au terme du cours, les participants maitrisent les différents contenus, si leurs compétences en recherche documentaire se sont développées, ils deviennent aptes à trouver par eux-mêmes les meilleurs outils et les ressources les plus adaptées à leurs besoins informationnels. Sur le plan pédagogique, What's up Doc est construit sur des activités pratiques de recherche, dans de vrais outils documentaires, à travers lesquelles on peut intégrer les éléments théoriques indispensables.

- Des exercices proposés dans chaque module permettent de mesurer les acquis et les améliorer, si nécessaire grâce aux retours théoriques.

- Des fiches de synthèse en format PDF (Que les participants peuvent sauvegarder ou imprimer à leur convenance) sont également associées à chaque module du cours.

- Des évaluations, toujours intégrées dans les modules, mobiliseront l'ensemble des compétences et permettront de mesurer la progression des participants. En outre, une évaluation finale mesurera si les participants ont réussi. Si ce n'est pas le cas, d'autres sessions du cours seront assurées, et autant d'occasions pour améliorer leurs résultats.

Les activités du MOOC What's up Doc se dérouleront sur un total de 8 semaines, soit une semaine par module ( 2 semaines pour le module 5 qui propose une activité participative aux apprenants, via le forum du cours), auxquelles seront ajoutées 2 semaines pendant lesquelles les participants peuvent participer au test final qui vérifiera s'ils ont réussi le cours.

Le temps de travail nécessaire pour réaliser les activités (théorie et exercices) est estimé à 2 heures par module.

Au niveau de l'évaluation du cours, le test final, composé de 22 questions, prend environ 30 minutes.

Les activités, les exercices et les quiz proposés dans les différents modules du cours sont des évaluations formatives qui visent à orienter et à vérifier le progrès des participants. Elles n'interviendront pas, cependant, dans l'évaluation finale, laquelle portera uniquement sur le test final du cours.

Le MOOC «Préparer et Réussir le DELF et le DALF » se déroulait en 6 semaines.

La séance 0 : Il s'agit d'un tronc commun qui permet aux participants de se positionner et d'évaluer leur niveau en français, pour savoir si vraiment ils ont intérêt à s'inscrire en DELF $\mathrm{B} 2$ ou en DALF C1. Pour les aider à choisir le niveau qui leur correspond, les enseignants proposent un sujet complet DELF du CIEP ainsi qu'un sujet DALF accompagnés de corrigés. Libres à tous les participants de les faire en entier ou pas, ou simplement d'y jeter un coup d'œil.

Le MOOC a pour objectif la préparation et l'entraînement aux épreuves spécifiques de chaque examen. En revanche, des liens sont communiqués pour permettre à ceux qui le désirent d'effectuer quelques exercices de remédiation en langue (orthographe et grammaire). Temps estimé : $2 \mathrm{H} 30$ pour $\mathrm{B} 2$ et $3 \mathrm{~h}$ pour $\mathrm{C} 1$ hebdomadaires. Après la séance 0 , les participants doivent s'inscrire dans un des 3 parcours. Les 4 semaines suivantes sont 
organisées autour des 4 domaines de compétences évalués aux examens, à savoir la $\mathrm{CO}, \mathrm{CE}$, $\mathrm{PE}$ et $\mathrm{PO}$. A chaque fois, ils disposent d'une petite vidéo de conseils méthodologiques.

Pour les épreuves de compréhension, l'évaluation est automatisée (c'est le logiciel qui leur attribue la note), sauf pour les questions ouvertes qui seront proposées en évaluation par les pairs munis d'un barème très précis pour leur faciliter la tâche.

La production écrite est également évaluée par les pairs (la production est notée par deux apprenants, la constitution des groupes étant du ressort du logiciel). Mais, là encore ils ont à leur disposition un corrigé et une grille d'évaluation.

Pour la production orale, la plate-forme là aussi constituera les groupes, les participants s'organisent, via un outil de communication en ligne (type Hangouts ou Skype) des simulations d'examen, toujours grâce à des pistes de réponse et une grille d'évaluation.

Le forum constitue un lieu d'échange et d'interactions entre les participants lors de l'activité collaborative. L'enseignant peut y participer mais ne répond pas systématiquement aux questions relatives au cours. Le forum servira à poster des productions écrites et à chercher collectivement des idées pour la production orale.

La séance 5 est un examen blanc complet avec 4 épreuves et leurs corrigés. Pour chaque séance et $r$ chaque compétence, 3 ou 4 sujets sont proposés avec des corrigés. Les participants ne sont pas tenus de les effectuer toute la même semaine. Mais pour obtenir l'attestation de suivi du MOOC, ils doivent faire au moins un nombre de sujets imposé et avoir obtenu 50\% de la totalité des points.

Nombre des participants : 700 participants

Le MOOC «Éducation par la recherche : smart city à l'École » a été pensé comme un espace d'inspiration et de co-construction pour répondre aux curiosités pédagogiques ou, de manière plus ambitieuse, pour accompagner les participants pas-à-pas dans la conception d'une maquette de projet reposant sur le modèle de l'Éducation par la recherche. Ainsi, ce MOOC propose de prototyper, seul ou à plusieurs, un futur projet de classe grâce aux ressources, aux activités et aux échanges avec les autres participants. Chacune des cinq semaines renvoie aux étapes possibles de mise en place et de réalisation d'un projet d'Éducation par la recherche :

1. Lancer le projet

2. Questions des élèves et état de l'art

3. Problématique et protocole de recherche

4. Mettre en place la recherche et traiter les données

5. Communiquer, publier, partager

Le MOOC se déroulait sur six semaines à partir de $11 \mathrm{~h} 00$ U.T.C. soit $12 \mathrm{~h} 00$ heures de Paris. Le pré-MOOC :

La période qui précède l'ouverture du MOOC intitulée semaine 0 avait pour objectif :

- de créer les premiers contacts avec les autres apprenants ;

- de nous informer sur les composants et les événements autour du MOOC et notamment sur son lancement (semaine 0) ;

- de nous offrir des premières ressources pour préparer notre entrée dans le MOOC ;

- de nous familiariser avec la plateforme FUN et avec la notion de parcours MOOC pour ceux qui découvrent ce format.

\section{Recueil de données sur les forums}

Le choix de ce modèle est dicté par sa capacité à démontrer, à travers l'analyse des conversations en ligne, la dynamique des échanges entre les apprenants et la nature des interactions en ligne. Ce modèle se base sur des notions que nous avons jugées adéquates pour l'analyse des interactions au sein des forums de discussion. D'une part, c'est une analyse de contenu inspirée par l'analyse conversationnelle ; nous allons y traiter les points de la 
structuration des échanges, du cadre participatif du forum et du format de production/réception des messages. D'autre part, une analyse séquentielle des échanges inspirée par l'analyse conversationnelle de Mandala [14] où nous allons marquer les aspects de la collaboration et la Co-construction, en nous appuyant sur les notions suivantes de l'analyse conversationnelle [14] - [15] :

- L'organisation des tours de parole ;

- L'alternance des tours de parole ;

- La notion de la paire adjacente ;

- Les types d'enchaînement entre les interlocuteurs ;

- Les accords/désaccords ;

- Les commentaires adressés ;

- La notion de réparation ;

\section{Traitement de données et analyse de corpus}

Pour ce qui est des forums de discussions [15], nous rappelons que nous avons procédé à la collecte des interactions dans les forums des trois MOOC. Notre intérêt pour la collaboration [16], la Co-construction des savoirs [17] - [18] et les interactions entre les participants nous ont guidés dans la sélection et la constitution du corpus. Ainsi, pour le MOOC What's up doc formation documentaire pour tous, nous avons conservé les interactions durant la semaine 2 et 3 ainsi que les 3 dernières semaines rassemblées dans un seul forum 7, 8 et 9. Pour le MOOC Smart city à l'école, nous avons gardé les interactions dans le forum de la semaine 3 et 4 rassemblées aussi au sein du même forum [19].

Pour le MOOC Préparer et réussir le DELF B2 et le DALF C1, compte tenu de la confusion des forums et des sujets de lancement, dès la première semaine, seul un forum considéré comme général est conservé. Ensuite, nous avons procédé à une observation préliminaire des sujets/thèmes dans chacun de ces forums en lisant les intitulés et le début des interactions. Concernant le MOOC What's up doc -formation documentaire pour tous, nous avons constaté que les sujets du forumde la semaine 2 portaient sur une discussion générale avec un nombre minime de contributions (8 interventions). Ils portaient également sur des sujets relatifs à des problèmes techniques ou sur le partage de certains documents et la constitution de groupes. Etantdonné que nous visons des fils de discussions où l'interaction, l'échange et le partage des idées et la collaboration sont présents, nous avons opté pour l'élimination de ce forum. La semaine 3 connaît presque les mêmes sujets à l'exception d'un forum discutant un thème initié par l'enseignant et donnant lieu à 25 contributions. Les semaines 7 et 8 ont porté sur 4 sujets différents dont chacuncorrespond à un forum unique. Là, nous nous sommes parvenus à une sélection par nombre de contributions. Nous avons alors conservé 2 forums ; débat 1 et 3 avec 22 et 31 contributions respectivement et avons éliminé les débats 2 (4 contributions) et 4 (2 contributions) [20].

En ce qui concerne le $M O O C$ Smart city à l'école, nous avons procédé également à une sélection par nombre de contributions. Ontété conservées les interactions autour d'un thème initié par l'enseignant qui renferment plus de 30 contributions. Par contre, trois autres de type général ou technique ont été éliminées étant donné qu'elles ne contiennent que des contributions qui varient en 1 et4 messages [20].

\section{Les solutions logicielles pour l'analyse de corpus}

La forte croissance de l'industrie de la langue a donné naissance à de nombreux programmes de traitement automatique du langage naturel. Ces derniers ont de nombreuses applications : reconnaissance et synthèse de la parole, formation de thésaurus et extraction 
de termes, interrogation de bases de données multilingues, traduction automatique, synthèse automatique, dialogue homme-machine, reconnaissance de langage, etc. Ces applications nécessitent différents niveaux d'analyse : acoustique, parole, phonétique, morphologie, syntaxe, sémantique et pragmatique. L'utilisation d'un corpus de grande taille nécessite l'utilisation de procédés de traitement automatisés. S'ils ne sont pas toujours totalement fiables, au moins ils ont l'avantage d'appliquer les principes de manière uniforme sur de grandes quantités de données, ce qui suffit à pallier les lacunes des traitements automatiques pour appliquer des techniques de vérification ou utiliser des procédures de traitement automatique contrôlées.

\section{L'analyse logiciel QDA miner}

QDA Miner est un logiciel d'analyse qualitative élaboré par une compagnie québécoise : Il semble de plus en plus utilisé en contextede recherche universitaire et rivalise avec d'autres logiciels mieux connus tel que N'Vivo. Quel que soit l'outil utilisé, la qualité d'une recherche qualitative n'est pas tant dépendante du logiciel que des compétences scientifiques de l'analyste. Autrement dit, QDA Miner aboutira à des résultats intéressants à condition de l'utiliser de manière pertinente au regard des objectifs spécifiques de chaque recherche.

\subsection{Résultats des données qualitatives}

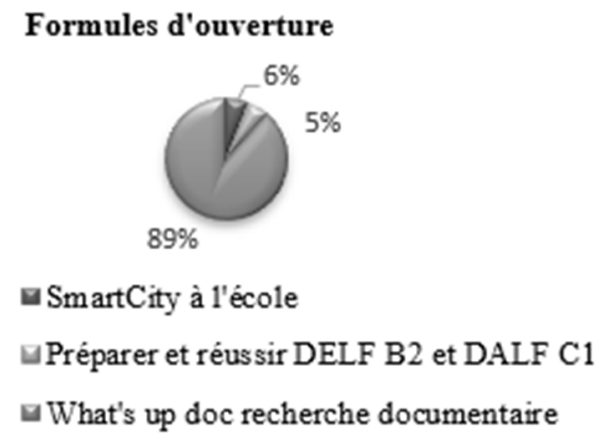

Fig. 1. Les formules d'ouverture de conversation sur les forums étudiés des trois MOOC.

Cette figure montre que le nombre de formules d'ouverture de conversation est élevé dans le MOOC What's up doc recherche documentaire $89 \%$. A l'opposé, le nombre est très bas pour les deux autres MOOC Smart City à l'école etPréparer et réussir le DELF B2 et le DALF Cl.



- What's up doc recherche documentaire

• Préparer et réussir DELF B2 et DALF C1

- SmartCity à l'école

Fig. 2. Le nombre de vouvoiement et tutoiement sur les forums étudiés des trois MOOC. 
Cette figure indique que le nombre de vouvoiement dans le MOOC What's up doc recherche documentaire qui est de 37 dépasseceux enregistrés dans le MOOC préparer et réussir le DELF $B 2$ et le DALF C1 (9) et dans le MOOC de Smart City à l'école (8). Concernant le tutoiement, cette figure montre une absence totale dans le MOOC de What's up doc recherche documentaire pour tous ( 0 tutoiement) et un score de 4 et de 1 , respectivement, pour les MOOC de Smart city à l'école et est de 4 avec un seul tutoiement au niveau du troisième MOOC Préparer et réussir le DELF B2 et le DALF C1.

\section{Alternance conversationnelle}

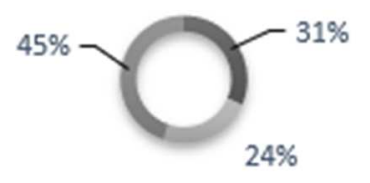

\section{- SmartCity à l'école \\ घréparer et réussir DELF B2 et DALF C1 \\ = What's up doc recherche documentaire}

Fig. 3. Le degré d'alternance des tours de parole.

L'analyse des données représentées dans cette figure expose le degré d'alternance des tours de parole au niveau des interactions surles forums étudiés. En règle générale, dans toute interaction, les tours de parole se succèdent sans problème, chaque participant ayant notamment pour tâche d'éviter les silences gênants (ceci signifie que chacun se doit de prendre la parole dès que l'autre a terminé son tour).

Cette figure nous apprend que :

- $\quad \mathbf{4 5 \%}$ d'alternance conversationnelle au niveau des interactions sur le forum du MOOC What's up doc

- $\quad 31 \%$ d'alternance conversationnelle au niveau des interactions sur le forum du MOOC Smart City à l'école.

- $\quad \mathbf{2 4 \%}$ d'alternance conversationnelle au niveau des interactions sur les forums du MOOC Préparer et réussir le DELF B2et le DALF C1.

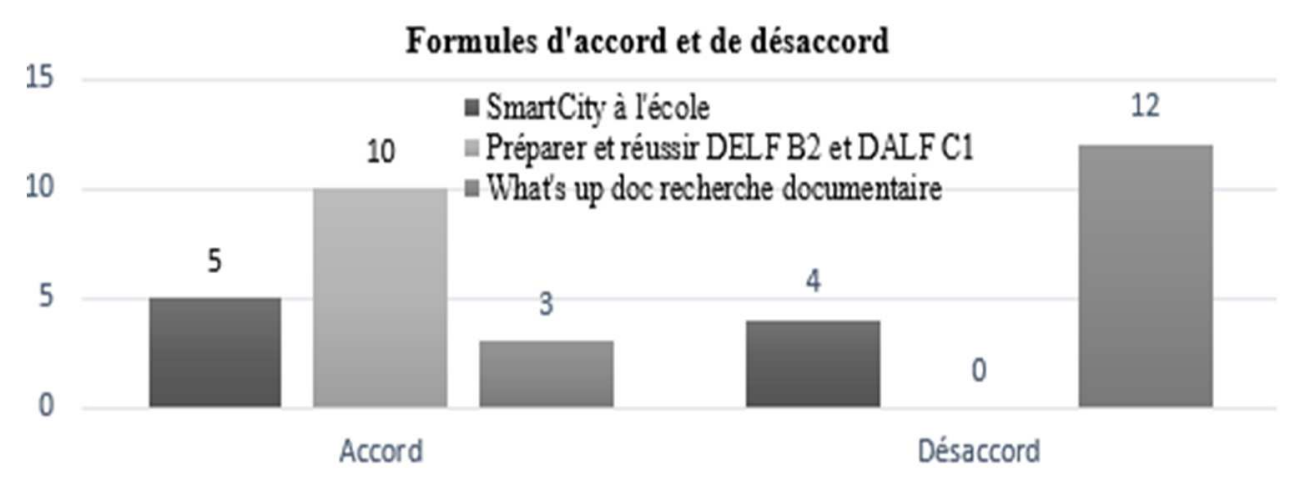

Fig. 4. Les types d'enchaînement entre les interlocuteurs. 
La présente figure indique le type d'enchaînement entre les interlocuteurs à travers la recherche des formules « d'accord et de désaccord ».

Par rapport au forum du MOOC préparer et réussir le DELF B2 et le DALF C1, les interlocuteurs affichent «L'accord » avec un nombre de 10. Quant au « Désaccord », il y est totalement absent.

En ce qui concerne le MOOC Smart City à l'école le nombre «d'accord et de désaccord» varie entre 4 et 5 . Quant au troisième MOOC, le nombre de désaccord entre les interlocuteurs sur le forum du MOOC what's up doc est élevé par rapport au 2 autres MOOC avec un nombre de 12.

\section{Formules de fermeture}

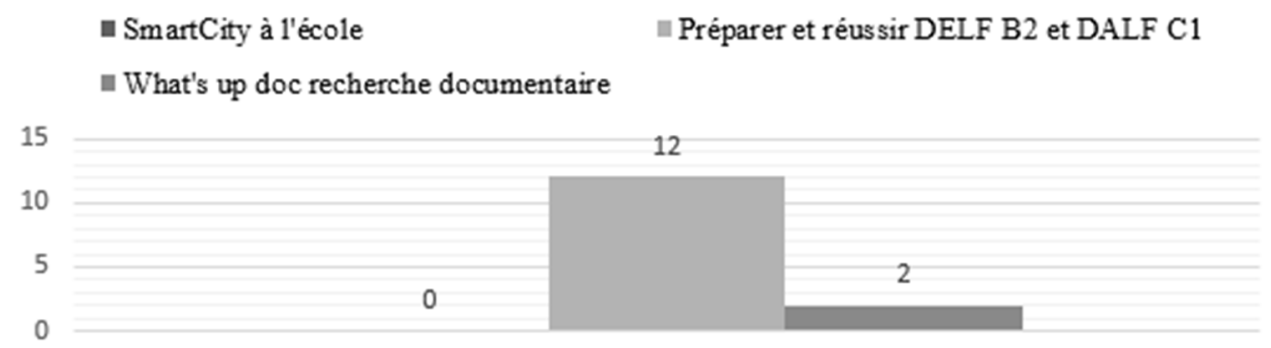

Fig. 5. Les formules de fermeture de conversation sur les forums étudiés des trois MOOC.

Cette figure montre que les formules de fermeture sont considérablement absentes sur le forum du MOOC Smart City à l'école. Sur le MOOC What's up doc recherche documentaire, les formules de fermeture sont très peu utilisées par les interlocuteurs (2) alors que sur le troisième MOOC préparer et réussir le DELF B2 et le DALF C1, elles sont bien présentes avec un total de 12 .

\subsection{Analyse des résultats}

Nous avons essayé de mettre en relief, tout au long de l'analyse qualitative de notre étude, les manifestations de la Co-construction du savoir dans les interactions en ligne à travers le modèle d'analyse conversationnelle d'inspiration ethnométhodologique.

Etant donné que l'archivage est une condition sine qua non dans une analyse conversationnelle pour les forums, nous avons dégagé trois formes, à savoir : un forum de consignes, un forum de présentation et de motivation et un forum de discussion. Il est à noter que c'est ce dernier qui est indispensable pour un apprentissage dans la mesure où les étudiants peuvent y discuter des sujets et enrichir ainsi leurs connaissances. Le rôle de l'enseignant demeure, cependant, primordial étant donné qu'il canalise les discussions et assurele débat. Plus l'interaction évolue, plus le rôle des apprenants et des enseignants évolue. L'intensité de l'interaction révèle la dynamique des échanges et la richesse de la discussion.

Il est intéressant de souligner, vu le très grand nombre de sujets initiés sans réponse parfois, que les participants du MOOC Prépareret réussir le DELF et le DALF considèrent le forum comme un espace de divertissement ou bien de discussion et non pas comme un lieu de construction des savoirs.

Quant au MOOC what's up doc, les participants sont engagés dans l'interaction et sont, en principe, tous des producteurs de messages.Ce sont des étudiants qui prennent part à la discussion, ajoutent des commentaires, réagissent aux propos des autres interactants et posent des questions qui suscitent de la discussion. L'ensemble des interventions est public et adressé normalement à tout le monde. Mais il existe des destinataires qui sont directement impliqués ce qui entraîne des conversations dyadiques ou triadiques [21]. 
Les participants au MOOC smart city à l'école, quant à eux, ont choisi dans la plupart des forums de ne pas interagir et d'être de simples observateurs. Nous pouvons appeler ce type d'étudiants les 'overhearers' en empruntant le terme utilisé par Goffman [21]. Les locuteurs sont conscients de la présence de ces personnes qui sont probablement en train de lire et d'observer les conversations mais sans aucun feedback.

Bref, de ce qui précède et à travers l'analyse des interactions en ligne dans les forums étudiés, il appert qu'il y a un taux plus au moins faible de coparticipation et Co- construction des savoirs au niveau des interactions en ligne entre les apprenants du MOOC Préparer et réussir le DELF et le DALF. Dans le cas des forums du MOOC What's up recherche documentaire, la situation est plus favorable ; les participants s'intéressent aux réponses de leurs pairs, accordent plus d'importance à leurs interventions et réagissent en conséquence.

Nous postulons que la faible présence dans le forum (MOOC Préparer et réussir le DELF et le $D A L F$ ) est due à la non compréhension de la notion de l'interaction en ligne. Les apprenants n'arrivent pas encore à saisir le rôle des interactions en ligne dans la construction collaborative des savoirs. De ce fait, nous jugeons nécessaire la clarification de ces points au lancement de la formation. Il s'agit de leur faire comprendre que le niveau de maîtrise de la langue ne doit pas être un obstacle ou une excuse à la non-participation. Ainsi, l'interaction doit être en vue du développement cognitif et sociocognitif et non pas en vue de l'évaluation finale.

En somme, à travers l'analyse des interactions en ligne au sein des forums étudiés, nous sommes parvenus à plusieurs constats surle degré d'interaction des apprenants en ligne. Dans un premier temps, les interactions dans le forum du MOOC 'Préparer et réussir le $D E L F$ et le $D A L F$ ' sont très faibles. Malgré la présence de presque tous les interactants, apparaît un manque de discussion, de collaboration et d'échange. Tout le monde répondait à la question posée par l'enseignant au départ ou par un participant sans enchainer avec les messages antérieurs ou répondre aux interventions de leurs amis. Il n'y avait aucun exemple de reprise de messages de la part des apprenants pour les commenter ou les évaluer.... Toutefois, tous recourent à des questions d'ordre technique ou relatives au dépôt des travaux. Soulignons que le cas de ce forum,en particulier, ne nous a pas permis d'apporter des éléments de réponse positive à notre troisième question de recherche vue l'absencede l'interaction entre les apprenants.

Nous avons dans un premier temps répondu à nos deux premières questions de recherche à travers l'analyse logiciel. Nous avons pu déduire à travers les forums que la collaboration est presque absente entre les participants pour des raisons diverses :

- Difficultés relationnelles et communicatives ;

- Désordre des forums ;

- Les sujets initiés sur les forums ne feront pas l'objet de la formation.

Aussi pouvons-nous conditionner cet apport du travail collaboratif en ligne par la nécessité d'assurer des conditions favorables à l'exemple d'un tuteur facilitateur de l'échange qui inciterait les apprenants à prendre part aux interactions.

La deuxième question de recherche porte sur le forum en tant que moyen qui facilite la collaboration et la communication entre lesapprenants.

La troisième question de recherche traitée lors de l'analyse qualitative a porté sur le rôle des interactions enligne dans la construction collaborative des savoirs dans les formations en ligne ?

Notre analyse des traces des étudiants dans les forums en premier lieu a permis de tirer la conclusion suivante : les apprenants n'ont pas encore saisi la notion des interactions en ligne et leur rôle dans la construction collaborative des savoirs. Leurparticipation collective reste généralement faible. Nous pensons également que la remédiation à cette situation passe par l'intervention de l'enseignant appelé à clarifier la notion de collaboration pour ses apprenants 
dès le début du cours. Ainsi, les apprenants doivent être sensibilisés quant au fait que les échanges ne doivent pas prendre place seulement pour des raisons d'évaluation finale ou bien pour combler le vide dans tel espaces de collaboration.

Les hypothèses formulées pour notre recherche ont porté sur l'adaptation d'une ingénierie pédagogique pour favoriser les interactions en ligne au service de la coconstruction du savoir. Notre analyse a permis de valider partiellement notre première hypothèse. Les interactions diffèrent d'un MOOC à l'autre. Dans certains cas, le forum représente un moyen de collaboration et de production à travers les échanges et les interactions entre les apprenants, mais dans d'autres cas, cela est moins favorable puisque l'interaction et le taux de participation demeurent faibles. Par conséquent, nous reprenons l'idée selon laquelle la position des apprenants vis-à-vis de l'usage des outils de collaboration ainsi que la manière dont ils les utilisent sont déterminantes. En d'autres termes, ces outils si bien utilisés par les apprenants peuvent constituer un vrai moyen de collaboration et de production en présence d'un enseignant accompagnateur, guide et facilitateur de l'échange. Par ailleurs, la deuxième hypothèse qui porte sur le rôle des forums dans la collaboration et la production n'a pas pu être validée.Les apprenants se trouvent encore timides et passifs dans ce genre d'espaces de collaboration dans les MOOC et gardent souvent la position de récepteurs qui ne prennent pas l'initiative d'animer. Ce qui va nous amène à rejeter la troisième hypothèse.

\section{Conclusion et perspectives}

L'objectif principal de cette recherche étant l'analyse de la construction collaborative des savoirs à travers les interactions en ligne sur les forums de discussion, l'étude s'est basée sur la description des aspects et des manifestations de la collaboration et la coparticipation dans les forums. Elle indique le degré de co-construction qu'il peut y avoir grâce aux forums qui permettent hcollaboration en ligne.

En réponse à notre problématique de recherche et sur la base des résultats obtenus, nous pouvons tirer les conclusions suivantes : Dans l'environnement et le contexte auxquels appartient notre public cible, c'est la manière d'utilisation des forums qui indique l'existence ou l'absence de la co-construction des savoirs. Cela dépend à notre avis de plusieurs facteurs :

- Le degré de motivation (intrinsèque et extrinsèque) des apprenants fait que certains interactants interviennent rarement, ce qui affecte le degré de collaboration et de coconstruction générale.

- Les attitudes des participants vis-à-vis de l'outil dans la mesure où certains n'ont pas l'habitude de créer des conversations sur des forums pédagogiques.

- La confusion entre le travail collaboratif et le travail coopératif affecte aussi le degré de la Co-construction.

- La maîtrise de la langue constitue un frein pour certaines personnes lors des interactions, ce qui explique pourquoi certains étudiants n'interviennent pas lors des échanges en ligne.

- Les scénarios de communication et d'encadrement adoptés sont un élément déterminant pour rassurer les apprenants en difficulté. Les résultats de notre étude ouvrent des perspectives de recherches qui portent essentiellement sur:

- La motivation des apprenants dans les espaces numériques de collaboration.

- Le non-usage des outils de collaboration et des forums dans les formations en ligne.

- Les problèmes linguistiques lors des interactions en ligne. Au terme de cette étude, nous espérons que les résultats obtenus retiendront l'attention des lecteurs et que ce travail servira de point de départ pour de futures recherches dans le domaine des interactions en ligne à des fins didactiques et pédagogiques. 


\section{Références}

1. Conseil de l'Europe. Cadre européen commun de référence pour les langues : apprendre, enseigner, évaluer (1- 196).Paris : Les Éditions Didier (2001).

2. H. Mandala, Etude des caractéristiques discursives et sociales de l'écrit numérique : de la communication écrite médiée à la co-construction interactive de l'écrit socionumérique. Thèse de doctorat, Linguistique. Université Aix-Marseille (2014).

3. B. Albero, \& P. Charignon, E-pédagogie à l'université : Moderniser l'enseignement ou enseigner autrement? Paris :Agence de mutualisation des universités et établissements (AMUE) (2008).

4. M. Marcoccia, (s.d), Polylogues en ligne : analyse conversationnelle des forums de discussion. Université de Technologie deTroyes, (2004).

5. L. Mondala, Formes de séquentialité dans les courriels et les forums de discussion. Une approche conversationnelle del'interaction sur Internet. Alsic, 2(1) (1999), pp.1-27. DOI : 10.4000/alsic. 1571.

6. M. Marcoccia, « L'analyse conversationnelle des forums de discussion : questionnements méthodologiques ». Les Carnets du Cediscor 8, pp. 1-12 (2004).

7. H. Blumer, "Social psychology", in E. P. Schmidt (dir.), Man and Society : A Substantive Introduction to the Social Science, Prentice-Hall, New York, 1937, pp. 144198

8. H. Garfinkel, Studies in ethnomethodology. Englewood-Cliffs, NJ: Prentice-Hall. (1967).

9. C. Kerbrat-Orecchioni. La notion d'interaction en linguistique : origine, apports, bilan. In: Langue française, $\mathrm{n}^{\circ} 117,1998$. La linguistique comme discipline en France, sous la direction de Jean-Louis Chiss et Christian Puech. pp. 51-67.

10. R. Vion, La communication Verbale. Analyse des interactions, Hachette Supérieur, Paris.

11. A.-N. Perret-Clermont, (1980). Social interaction and cognitive development in children.London: Academic Press. (Traduction en anglais de La construction de l'intelligence dans l'interaction sociale). Peter Lang, (1979).

12. j. Lave, Situated Learning. Legitimate Peripheral Participation, Cambridge, Cambridge University Press (1991).

13. I. Strioukova, L'usage de forum de discussion comme une pratique située dans le cadre d'une FOAD. Communication présentée au Colloque Innovation (2006).

14. L. Mondala, Analyser les interactions en classe : quelques enjeux théoriques et repères méthodologiques. Université deLausanne et Neuchâtel : TRANEL 22, pp. $55-89$ (1995).

15. F. Henri, B. Charlier, «L'analyse des forums de discussion pour sortir de l'impasse » pp. 1-8 (2004).

16. F. Henri, K. Lundgren-Cayrol, Apprentissage collaboratif et nouvelles technologies (1191). Bureau des technologies d'apprentissage : centre de recherche LICEF (1998).

17. F. Le Menaheze, Coopérer pour apprendre! La coopération entre élèves, la médiation de l'enseignant : un processus de co-construction des savoirs. (Mémoire de maîtrise). Université de Nantes, (2002).

18. H. Mandala, Etude des caractéristiques discursives et sociales de l'écrit numérique : de la communication écrite médiée à la co-construction interactive de l'écrit socionumérique. Thèse de doctorat, Linguistique. Université Aix-Marseille (2014). 
19. F. Mangenot, « Analyser les interactions pédagogiques en ligne, pourquoi, comment?» Dans J, Gerbault, La langue ducyberespace : de la diversité aux normes, Paris : L'Harmattan, pp. 1-11 (2007).

20. L. Mondala, Analyser les interactions en classe : quelques enjeux théoriques et repères méthodologiques. Université de Lausanne et Neuchâtel : TRANEL (22), pp. 55-89 (1995).

B. Céline, "Goffman et l'ordre de l'interaction : un exemple de sociologie compréhensive », Philonsorbonne [En ligne], 1 |, misen ligne le 20 janvier 2013, consulté le 10 novembre 2020. DOI : https://doi.org/10.4000/philonsorbonne.102. (2007). 